\title{
A Secure Test Technique for Pipelined Advanced Encryption Standard*
}

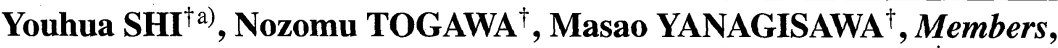 \\ and Tatsuo OHTSUKI ${ }^{\dagger}$, Fellow
}

SUMMARY In this paper, we presented a Design-for-Secure-Test (DFST) technique for pipelined AES to guarantee both the security and the test quality during testing. Unlike previous works, the proposed method can keep all the secrets inside and provide high test quality and fault diagnosis ability as well. Furthermore, the proposed DFST technique can significantly reduce test application time, test data volume, and test generation effort as additional benefits.

key words: scan test, security, test quality

\section{Introduction}

The rapid growth of communication systems results in an increasing demand for powerful cryptographic techniques. As a consequence, the availability of hardware that supports cryptographic algorithms is becoming increasingly critical. Many cryptographic algorithms, such as Data Encryption Standard (DES) [1], Advanced Encryption Standard (AES) [2], Rivest Shamir Adleman (RSA) [3], etc. have been implemented as application-specific integrated circuits (ASICs) [4], [5], or as cryptographic coprocessors [6], [7] due to the increasing requirement on high-throughput encryption.

To ensure the correctness of crypto hardware, it is always desirable to conduct testing at fabrication and in-field, which is because without adequate test coverage, the manufacturer can not be assured of the product's quality. To achieve high test quality, scan test is the most powerful and widely adopted design-for-test (DFT) technique for sequential circuits. Nowadays, almost all of the current design-fortest (DFT) techniques start with a baseline of scan technology. Unfortunately, scan chains would be used to discover the internals of crypto hardware, which presents a significant security risk of information leakage. Recently, it has been reported that scan chains can be used as a "side channel" to recover the secret keys from a hardware implementation of any cryptographic algorithm [8]. In cryptology this problem is well known as side channel cryptanalysis. Thus, to achieve high test quality by increasing the testability of the CUT and to maintain the security appears to be conflict with each other. Under such contradictory requirements, to

Manuscript received April 9, 2007.

Manuscript revised August 10, 2007.

${ }^{\dagger}$ The authors are with the Faculty of Science and Engineering, Waseda University, Tokyo, 169-8555 Japan.

${ }^{*}$ This paper was presented at ISCAS2007.

a)E-mail: shi@yanagi.comm.waseda.ac.jp

DOI: 10.1093/ietisy/e91-d.3.776 develop efficient test strategies for crypto hardware becomes an emergent task.

Although it has been agreed in the industry that scan technology carries the potential of being misused to leak out the secret information, there was not much work done related to the security of scan test before [8]. One common straightforward method is to blow the test circuitry after manufacturing test. In this way, after manufacturing test, the test circuitry can only be accessible by few or a few restrictive end-users so that it can prevent the internal scan chains being misused to retrieve the secret information. Nowadays, such techniques have been broadly used in the smartcard application, however it is not an ideal solution because it will compromise the maintenance and debug capabilities in-field. In [9], flip-flops that contain secret information are included in a special scan chain. This scan chain is only accessible by chip manufacturers but not by customers. However, in a stand-alone crypto hardware, almost all of the flip-flops could contain information related to a secret. This architecture also limits the maintenance and debug features of the crypto core in a system. There are also some works focused on using built-in self-test (BIST) such as that in [10]. BIST may be a promising solution, because it does not require visible scan chains, but BIST incurs more overhead and yields less fault coverage when compared to scan test within limited test time. On the other hand, fault diagnosis becomes another problem with BIST. In [11], a scan chain scrambling technique was proposed to provide both security and testability for crypto hardware. This method dynamically reorders the flip-flops in a scan chain to make it more difficult for the hacker to discover the internal scan architecture. However, statistical analysis of the information scanned out from chips can still determine the scan chain structure and the secret information. Furthermore, the area overhead incurred by dynamic reordering of internal scan chains is high and also make it difficult for fault diagnosis. This is because each section of the dynamic configured scan chain need to be followed with a n-to-1 multiplexer where $n$ is the number of sections in the whole scan chain. In [12], an implementation of mirror key registers (MKR) that could separate the secret key from the scan chain was proposed, and a on-chip test controller was designed to limit the crypto hardware being switched between scan test mode and the normal mode. However, to specify MKRs to corresponding secret key bit requires secure synthesis and testing of MKRs is not straight-forward. Meanwhile, in [12] where 
two copies of secret keys are required, although mirror keys are used during scan test, it still carries the potential of being combined with Differential Power Analysis (DPA) to compute the secret information. In addition, it could not support fault diagnosis and on-line testing.

To address this problem, in this paper we discuss the development of a design-for-secure-test (DFST) technique for pipelined AES as a case study. The basic idea of the proposed method is to KEEP ALL THE SECRET INSIDE not only in scan test mode but also in diagnosis mode. Unlike previous works, the significant advantages of the proposed secure scan DFT architecture are four folds:

- High Security - Any information obtained from scan out wouldn't be used to retrieve the secret keys or any hided information.

- High Test Quality - Crypto hardware could be fully tested using scan-based test technology.

- Pass/Fail test and excellent diagnostics capability.

- Low hardware overhead and significantly reduction in test application time, test data volume and test generation effort.

The rest of this paper is organized as follows: Section 2 illustrates the proposed design-for-secure-test (DFST) architecture for high-throughput pipelined AES implementations. Sections 3 and 4 present the experimental analysis and conclusions, respectively.

\section{Proposed Approach}

In this section we will illustrate the proposed DFST technique for pipelined AES. Meanwhile, fault diagnosis is a very important test issue when developing DFT techniques. Unfortunately, as far as we know, by now no work has been conducted considering security, testability and fault diagnose ability together. Thus we then attempted to refine the DFST architecture to incorporate diagnosis ability without any secret information leakage during diagnosis as well as during test.

\subsection{DFST Architecture}

As introduced above, when developing test techniques for crypto hardware, not only test issues but also security should be taken into account. Thus, an application-specific test technique seems to be the right solution for crypto hardware. In our work, we developed a DFST technique for pipelined Rijndael AES circuit as a representative to show how we can use scan test technique to achieve high test quality without any compromise of internal security.

Rijndael AES is generally composed of four different steps that are repeated in $N_{r}$ number of rounds. These are byte substitution, shift row, mix column, and key addition. When a key size of 128 bits is used, the number of rounds $\left(N_{r}\right)$ is equal to 10 . Figure 1 shows the pipelined implementation of Rijndael AES algorithm [6]. The shift row step is

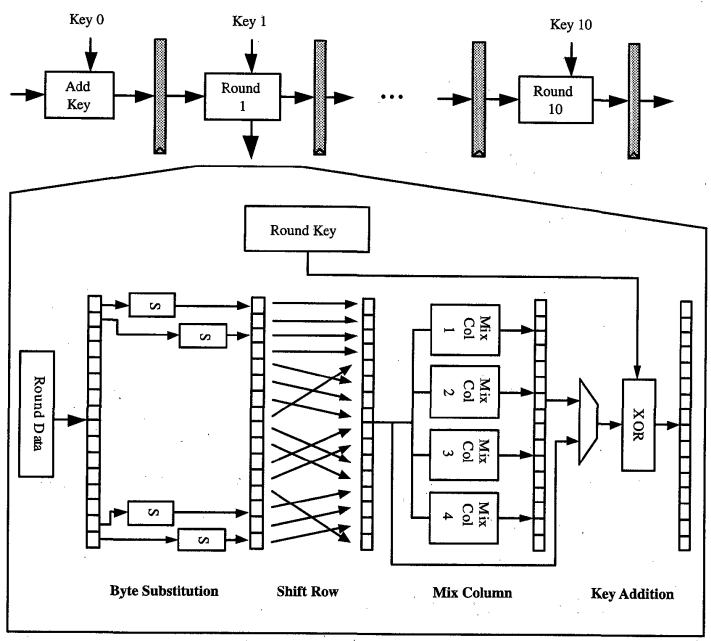

Fig. 1 Pipelined AES from [6].

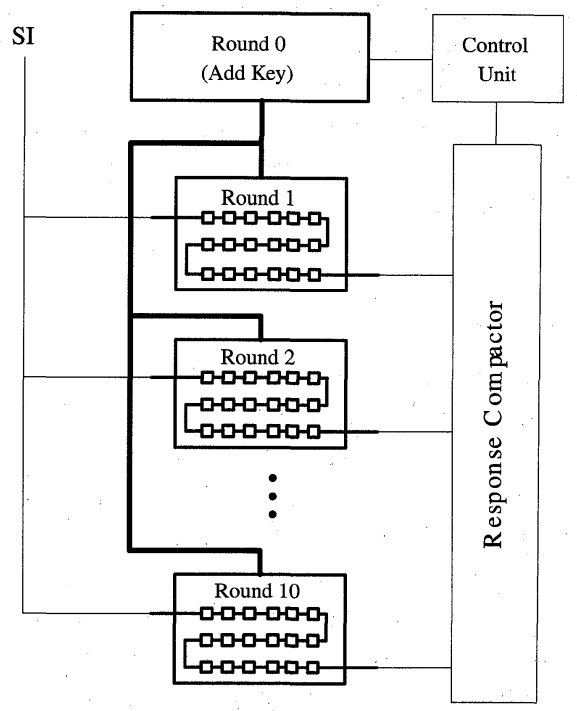

Fig. 2 Proposed test structure.

just an interconnection and the key addition is a XORing result of the round data and the round key. The mix column step consists of a chain of XORs to permute the elements of data in each column. In a pipelined AES [6], the one-round module is replicated 10 times and each round has a different round key. Although the last round does not has a mix column operation, it is reasonable that all of the 10 rounds have the same module. The output of each round is registered and applied to the next round. Our work is based on such a model of pipelined AES. Furthermore, we assume that the round keys are precomputed from the user key and stored in the on-chip RAM and during test the same key (all zero) is loaded.

The proposed DFST structure is shown in Fig. 2, which mainly consists of a response comparator. Since in the pipelined AES, the 10 round modules are identical, they should have the same scan chain architecture. Thus in our work the identified scan chain of each round module are tied 


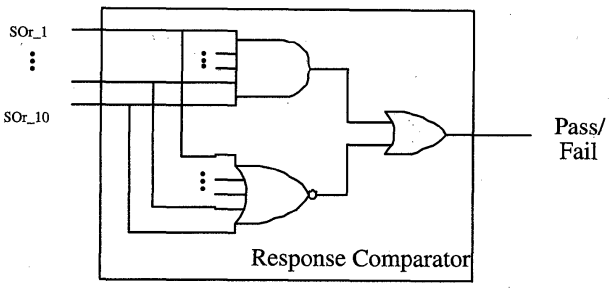

Fig. 3 Response comparator architecture.

together and driven using a common test channel. The output responses from the scan chain of each round module are fed into the comparator. Since the round modules of the modeled AES are identical, therefore when there is no manufacturing defect, the scan outputs of all the round module should be same. The comparator is used to compare the outputs from each module. Figure 3 shows the implementation of the comparator. Because each corresponding output from each round module should be compared, it only requires a 10-input NOR gate, a 10-input AND gate and a 2-input OR gate. If the responses from all the 10 rounds are identical, the comparator outputs the pass signal; otherwise a fail signal is outputted to indicate that at least one of the round modules fails the test.

\subsection{Refined for Diagnosis}

From the test point of view, it would always be desirable that test technique should incorporate fault diagnosis so as to correctly identify the defect inside the circuit and then improve the manufacturing process. For full-scan based designs, it is generally agreed that scan test can enable efficient fault diagnosis by providing direct access to the internal nodes of the circuit. However in the proposed architecture, the output responses are compacted into a signature, it only provides pass/fail information and contains very little diagnostic information. Therefore we improve the above architecture to increase fault diagnosis ability without any compromise of security and test quality.

To run fault diagnosis, we should identify the failing vectors and the corresponding mismatch scan cells. Since in the proposed architecture it is easy to identify the failing vectors, in our work we focus on how to determine the mismatched scan chains (i.e. the scan cells). The refined response comparator logic is shown in Fig. 4. In Fig. 4, instead of only generating the pass/fail signal, a $N_{e r r}$ is also generated using only XOR gates, which is XORing all the scan responses from all the round modules. The $N_{e r r}$ signal can be used to indicate the number of the mismatched responses. Since $N_{e r r}$ is XORing the responses, when it equals to 0 , it indicates that the number of mismatched responses is even, otherwise the number of mismatch responses is odd as shown in Table 1. To do this is very useful, because it can provide available information for fault diagnosis.

Here let us explain the diagnosis procedure to identify the mismatched scan cells. During diagnosis, the test control logic is set to diagnosis mode first. Then the identified fail-

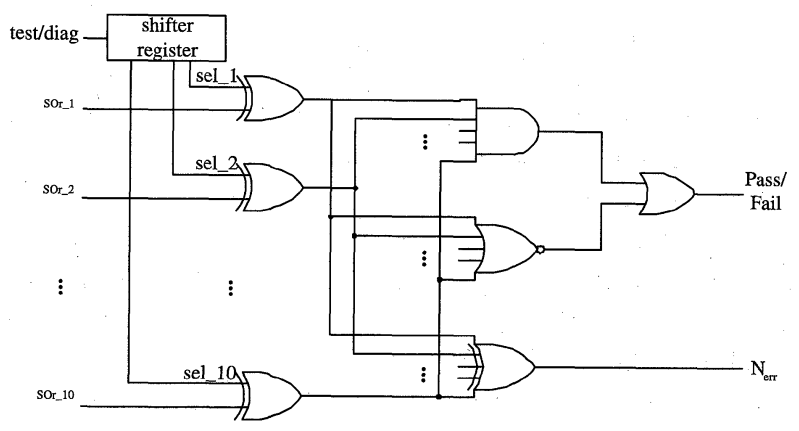

Fig. 4 Refined response comparator.

Table 1 Comparator outputs.

\begin{tabular}{c|c||c}
\hline \hline Pass/Fail & $N_{\text {err }}$ & Meaning \\
\hline Pass & 0 & No defects \\
\hline Fail & 0 & Defects exist and the number is even \\
\hline Fail & 1 & Defects exist and the number is odd \\
\hline \hline
\end{tabular}

ing vector is applied and test responses are captured. The sel signal is generated on-chip under the control of test/diag signal, which is not controlled from the external. In our work, we simply implemented it using a shifter register. The sel signal is used to select the corresponding round module (i.e. the scan chain) for test and diagnosis. In scan test mode, the sel signal is set to all zeros " 0000000000 ", which indicates that the entire scan responses are shifted into the response comparator in parallel as it is. In diagnosis mode, for a failing vector, the sel signal is generated as from "0000000001" to " 1000000000 ", which means the scan responses of the 10 round modules are individually selected to be inverted before entering into the comparator.

Let us look at an example. If a scan response is "1111111111", then the response comparator outputs a "Pass" signal and $N_{e r r}$ signal is '0', which means there is no error response. When the scan response is "1111111011", then the response comparator outputs a "Fail" signal and $N_{\text {err }}$ signal is ' 1 '. In this case, it means there exists a mismatched response(s) in the scan chains, and the number of mismatch responses is odd. Thus, when we enter the diagnosis mode, the sel signal is first generated as "000000001", which means the response from the first round module is inverted before entering into the comparator; then the response comparator outputs the "Fail" signal and the $N_{e r r}$ signal is ' 0 '. From these two signals, we can learn that the first round module has no defect. Then we shift the same vector into the circuit and then the sel signal would be "0000000010", "0000000100" and so on until the response comparator outputs the "Pass" signal and the $N_{\text {err }}$ signal equals to ' 0 '. By this means we can identify the mismatch scan cells in fault diagnosis without any secret information leaked out of the circuit. Here it should be mentioned that using the proposed DFST architecture we can only detect one error among the scan chains in limited time without any secret leakage. Future work will be concentrated on how to detect multiple errors during fault diagnosis. 


\section{Analysis and Discussion}

In this section, we present the experimental results on a pipelined AES implementation to show the effectiveness of the proposed DFST method and discuss how our approach is resistant to scan-based side channel attack.

\subsection{Resistance to SSCA}

To validate the effectiveness of the proposed DFST technique, we will discuss how the proposed architecture is resistant to scan-based side channel attack (SSCA). In SSCA presented in [8], one of the basic assumptions is that the internal secret information could be scanned out of crypto hardware, so that hackers may use it to determine the scan chain architecture of the crypto hardware and reveal the secret keys. However, in our method, no matter what the scan contents are, the scan out is always Pass or Fail, which does not indicate the value of any internal scan cells or the data from any scan inputs. On the other hand, no matter what scan inputs are, the scan output will never be correlated with the scan inputs; thus even with statistical analysis, neither the internal scan architecture nor the key related information could be revealed by hackers.

\subsection{Implementation Analysis}

To analysis the overhead of the proposed DFST technique and to validate its efficiency, we incorporated it into a welldesigned pipelined AES implementation and the results are shown in Table 2. In our work we assume that the secret keys are stored in non-volatile memory. The AES was implemented in VHDL and synthesized using Synopsys Design Compiler to examine the hardware overhead of the proposed DFST architecture. The area overhead of the conventional full scan based AES is in 326K equivalent gates count with $2.6 \mathrm{~K}$ scanable flip-flops, when using the proposed method the addition hardware overhead is negligible, because there is only 62 additional gates mainly due to the response comparator. In addition, the proposed DFST architecture does not degrade the AES performance. Since only one additional MUX is inserted on the critical path for the scanable flip-flop, the delay is negligible.

In our method, the scan chains were designed so that multiple instantiations of AES round operation got identical chains. This helps in generating ATPG patterns by making use of scan equivalent structures. It also simplified pattern development by swapping patterns for identical chains to give coverage for all the repeated units. In our work,

Table 2 Experimental analysis.

\begin{tabular}{c|c|c|c|c}
\hline \hline Circuits & Measurement & Full-scan & DFST & Impr. \\
\hline \multirow{3}{*}{$\begin{array}{c}\text { Pipelined } \\
\text { AES }\end{array}$} & Area Overhead & $6.8 \%$ & $6.8 \%$ & 0 \\
\cline { 2 - 5 } & Fault Coverage & $100 \%$ & $99.9 \%$ & $-0.1 \%$ \\
\cline { 2 - 5 } & ATPG time & $>26 h$ & $38 \mathrm{~m}$ & $40 \mathrm{X}$ \\
\cline { 2 - 5 } & Test Data & $238 \mathrm{~K}$ & $28 \mathrm{~K}$ & $8.5 \mathrm{X}$ \\
\hline \hline
\end{tabular}

we insert a scan chain for the AES round module, and link them together at the top level. So from the top AES module, 10 identical scan chains exist in the design. In addition, in test mode, each round inputs are driven by the outputs of the preround. And then we use a commercial ATPG tool to generate test patterns for the pipelined AES and made a comparison between the conventional full scan method and the proposed round scan DFST method. It should be mentioned here that in the experiment results, the fault coverage of DFST contains not only the original data path of AES but also the introduced test circuit. In our method, a few nonscan FFs are inserted into the designs, which include some flops in the Test Control Unit (TCU) and the flip-flops of the refined response compactor as shown in Fig. 4. In addition, due to the security consideration, the AND gates and NOR gates in the proposed response compactor would lead to some fault coverage loss. So the total fault coverage of our method is $99.9 \%$. We can also find that when compared with full scan, the ATPG time is shorten by a factor of about $40 \mathrm{X}$ and the total test data volume is reduced to $12 \%$.

The proposed DFST architecture is the most suitable test technique for pipelined AES. Although it is applicationspecific, it has a lot of advantages over full scan or other existing DFT techniques for crypto hardware. It can provide high security as BIST, high fault coverage and fault diagnosis ability in the presence of only one error among all the scan chains, and is not susceptible to X states, as the effect of $\mathrm{Xs}$ is not accumulated, with negligible hardware overhead. In addition, test data volume is also greatly reduced.

\section{Conclusions}

Scan chains can be used as a "side channel" to recover secret keys from a crypto hardware implementation. Thus, testability and security seems to be contradicted with each other. However due to the requirement of high test quality, we can't eliminate scan chains, but to incorporate security into DFT. In this paper, we have proposed a DFST technique as a countermeasure to prevent secret information being leaked out of crypto hardware during test. Unlike previous works, we took security, test quality and fault diagnosis into consideration. Although the proposed DFST technique is an application specified method, it can provide high security as BIST, high fault coverage and fault diagnosis as full scan, and great test data compression with negligible hardware overhead. As far as we know, this is the first attempt to integrate security, testability and fault diagnosis ability together in crypto hardware testing.

\section{Acknowledgments}

This work was partially supported by Japan Society of the Promotion of Science (JSPS) under Grants-in-Aid for Young Scientists (B) (No.19700045). 


\section{References}

[1] National Bureau of Standards, Data Encryption Standard (DES), Federal Information Processing Standards Publication 46, 1977.

[2] National Institute of Standards and Technology (U.S.), Advanced Encryption Standard (AES). http://csrc.nist.gov/publication/drafts/ dfips-AES.pdf

[3] R. Rivest, A. Shamir, and L. Adleman, "A method for obtaining digital signatures and public-key cryptosystems," Commun. ACM, vol.21, no.2, pp.120-126, Feb. 1978.

[4] M. Aigner, S. Mangard, and S. Dominikus, "A highly regular and scalable AES hardware architecture," IEEE Trans. Comput., vol.52, no.1, pp.483-491, April 2004.

[5] N.S. Kim, T. Mudge, and R. Brown, "A $2.3 \mathrm{~Gb} / \mathrm{s}$ fully integrated and synthesizable aes rijndael core," Proc. IEEE Custom Integrated Circuits Conference (CICC), pp.193-196, May 2003.

[6] B. Lai, K. Tiri, A. Hodjat, D. Hwang, and I. Verbauwhede, "A 3.84 gbits/s AES crypto coprocessor with modes of operation in a $0.18-\mu \mathrm{m}$ CMOS technology," Proc. ACM Great Lakes Symposium on VLSI (GVLSI), pp.60-63, April 2005.
[7] I. Verbauwhede, P. Schaumont, and H. Kuo, "Design and performance testing of a $2.29-\mathrm{GB} / \mathrm{s}$ rijndael processor," IEEE J. SolidState Circuits, vol.38, no.3, pp.569-572, March 2003.

[8] B. Yang, K. Wu, and R. Karri, "Scan based side channel attack on dedicated hardware implementations of data encryption standard," Proc. IEEE International Test Conference (ITC), pp.339-344, Oct. 2004.

[9] R. Easter, E. Chencinski, E. D'Avignon, S. Greenspan, and C. Norberg, "S/390 parallel enterprise server CMOS cryptographic coprocessor,” IBM J. Res. Dev., vol.43, no.5/6, pp.761-776, 1999.

[10] H. Bonnenberg, H. Kaeslin, N. Felber, R. Zimmermann, A. Curiger, and $\mathrm{W}$. Fichtner, "A $177 \mathrm{Mb} / \mathrm{sec}$ VLSI implementation of the internation data encryption algorithm," IEEE J. Solid-State Circuits, vol.29, no.3, pp.303-307, May 1994.

[11] D. Hely, F. Bancel, M.L. Flottes, B. Rouzeyre, M. Renovell, and N. Berard, "Scan design and secure chip," Proc. IEEE International On-Line Testing Symposium (IOLTS), pp.219-226, July 2004.

[12] B. Yang, K. Wu, and R. Karri, "Secure scan: A design-for-test architecture for crypto chips," Proc. ACM/IEEE Design Automation Conference (DAC), pp.135-140, June 2005. 